\title{
The transverse momentum distribution of the Higgs boson at the LHC*
}

\section{Massimiliano Grazzini}

INFN, Sezione di Firenze, I-50019 Sesto Fiorentino, Florence, Italy

E-mail: grazziniefi.infn.it

\begin{abstract}
We present QCD predictions for the transverse momentum $\left(q_{T}\right)$ distribution of the Higgs boson at the LHC. At small $q_{T}$ the logarithmically-enhanced terms are resummed to all orders up to next-to-next-to-leading logarithmic accuracy. The resummed component is consistently matched to the next-to-leading order calculation valid at large $q_{T}$. The results, which implement the most advanced perturbative predictions available at present for this observable, show a good stability with respect to theoretical uncertainties. The numerical program $H q T$, used to perform the calculation, is briefly discussed.
\end{abstract}

International Europhysics Conference on High Energy Physics

July 21st - 27th 2005

Lisboa, Portugal

${ }^{*}$ Work done in collaboration with S. Catani, G. Bozzi and D. de Florian. 
The search for the Higgs boson is one of the highest priorities of the LHC physics program [1]. In the last years a significant effort has been devoted to refining the theoretical predictions for the various Higgs production channels and the corresponding backgrounds, which are now known to next-to-leading order accuracy (NLO) in most of the cases [2]. In the case of gluon-gluon fusion, which is the main Standard Model Higgs production channel, even next-to-next-to leading order (NNLO) QCD corrections have been computed, although in the large- $M_{t}$ approximation ( $M_{t}$ being the mass of the top quark). The result has been obtained first for the total rate [3], and more recently for fully exclusive distributions [4]. Among the possible observables, an important role is played by the transverse-momentum spectrum of the Higgs boson, whose knowledge may help to enhance the statistical significance of the signal over the background.

When the transverse momentum $q_{T}$ of the Higgs boson is of the order of its mass $M_{H}$, the perturbative series is controlled by a small expansion parameter, $\alpha_{\mathrm{S}}\left(M_{H}^{2}\right)$, and the fixed-order prediction is reliable. The leading order (LO) calculation [5] shows that the large- $M_{t}$ approximation works well as long as both $M_{H}$ and $q_{T}$ are smaller than $M_{t}$. In this framework, the NLO QCD corrections have been known for some time [6, 7, 8, 4].

The small- $q_{T}$ region $\left(q_{T} \ll M_{H}\right)$ is the most important, because it is here that the bulk of events is expected. In this region the coefficients of the perturbative series in $\alpha_{\mathrm{S}}\left(M_{H}^{2}\right)$ are enhanced by powers of large logarithmic terms, $\ln ^{m}\left(M_{H}^{2} / q_{T}^{2}\right)$. To obtain reliable perturbative predictions, these terms have to be systematically resummed to all orders in $\alpha_{\mathrm{S}}$ [9]. In the case of the Higgs boson, the resummation has been explicitly worked out at leading logarithmic (LL), next-to-leading logarithmic (NLL) [10], [11] and next-to-next-to-leading logarithmic (NNLL) [12] level. The fixed-order and resummed approaches then have to be consistently matched at intermediate values of $q_{T}$, so as to avoid double counting.

In the following we present predictions for the Higgs boson $q_{T}$ distribution at the LHC within the formalism of Refs. [13]-[15]. In particular, we include the best perturbative information that is available at present: NNLL resummation at small $q_{T}$ and NLO calculation at large $q_{T}$.

An important feature of our formalism is that a unitarity constraint on the total cross section is automatically enforced, such that the integral of the spectrum reproduces the known fixed-order results. More details are given in Ref. [15]. Other phenomenological results can be found in Ref. [16].

We now present quantitative results from Ref. [15] at NLL+LO and NNLL+NLO accuracy. At NLL+LO (NNLL+NLO) accuracy the NLL (NNLL) resummed result is matched to the LO (NLO) perturbative calculation valid at large $q_{T}$. Our calculation is implemented in the numerical program $\mathrm{HqT}$, which can be downloaded from [17]. The code is an improved version of the original program used in Ref. [14], the main difference being in the matching procedure, which is now performed using the results of Ref. [8].

The numerical results in Figs. 1 and 2 are obtained by choosing $M_{H}=125 \mathrm{GeV}$ and using the MRST2004 set of parton distributions [18]. At NLL+LO, NLO parton densities and 2-loop $\alpha_{\mathrm{S}}$ are used, whereas at NNLL+NLO we use NNLO parton densities and 3-loop $\alpha_{\mathrm{S}}$. The NLL+LO results at the LHC are shown in Fig. 1. In the left panel, the full NLL+LO result (solid line) is compared with the LO one (dashed line) at the default scales $\mu_{F}=\mu_{R}=M_{H}$. We see that the LO calculation diverges to $+\infty$ as $q_{T} \rightarrow 0$. The finite component, obtained through the matching procedure, is also shown (dotted line). The effect of the resummation, relevant below $q_{T} \sim 100 \mathrm{GeV}$, leads to a 

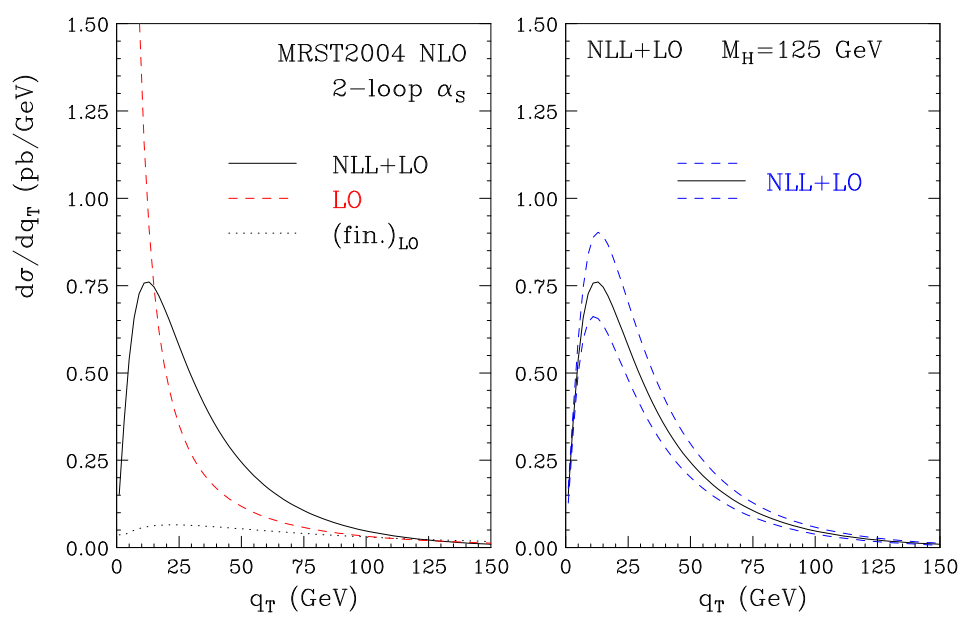

Figure 1: $L H C$ results at $N L L+L O$ accuracy.

physically well defined distribution at $q_{T} \rightarrow 0$. In the right panel we show the NLL+LO band obtained by varying $\mu_{F}$ and $\mu_{R}$ simultaneously and independently between $0.5 M_{H}$ and $2 M_{H}$, imposing $0.5 \leq \mu_{F} / \mu_{R} \leq 2$. The integral of the spectrum agrees with the total NLO cross section to better than $1 \%$. The corresponding NNLL+NLO results are shown in Fig. 2. In the left panel, the full
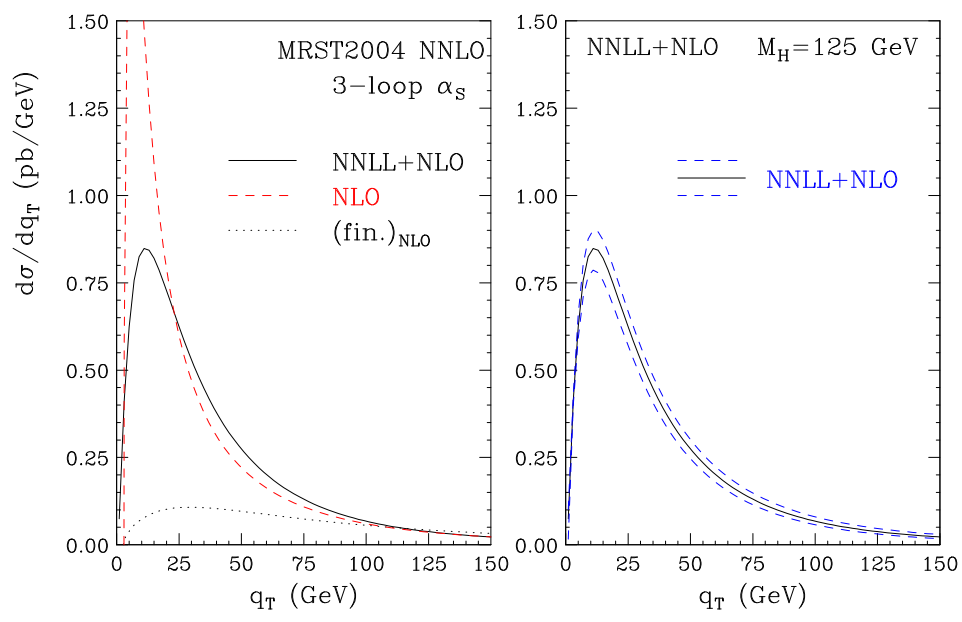

Figure 2: $L H C$ results at $N N L L+N L O$ accuracy.

result (solid line) is compared with the NLO one (dashed line) at the default scales $\mu_{F}=\mu_{R}=M_{H}$. The NLO result diverges to $-\infty$ as $q_{T} \rightarrow 0$ and, at small values of $q_{T}$, it has an unphysical peak that is produced by the numerical compensation of negative leading and positive subleading logarithmic contributions. The finite component (dotted line) vanishes smoothly as $q_{T} \rightarrow 0$, showing the quality of our matching procedure. The NNLL+NLO resummed result is slightly harder than the NLL+LO one, and its integral is in very good agreement with the NNLO total cross section. The right panel of Fig. 2 shows the scale dependence computed as in Fig. 1. Comparing Figs. 1 and 2, 
we see that the NNLL+NLO band is smaller than the NLL+LO one and overlaps with the latter at $q_{T} \lesssim 100 \mathrm{GeV}$. This suggests a good convergence of the resummed perturbative expansion. Other sources of perturbative uncertainty give smaller effects [15].

In summary, considering the above results, the perturbative uncertainty of the NNLL+NLO spectrum is of about $10 \%$ at intermediate and small $q_{T}$, where the bulk of the events is concentrated. At very small $q_{T}\left(q_{T} \lesssim 10 \mathrm{GeV}\right)$ non-perturbative effects should be taken into account, whereas at large $q_{T}$ the perturbative uncertainty increases. Our results for the $q_{T}$ spectrum are thus fully consistent with those on the total NNLO cross section [3].

\section{References}

[1] CMS Coll., Technical Proposal, report CERN/LHCC/94-38 (1994); ATLAS Coll., ATLAS Detector and Physics Performance: Technical Design Report, Vol. 2, report CERN/LHCC/99-15 (1999).

[2] Proceedings of the Workshop on Physics at TeV Colliders, Les Houches, France, 2001, hep-ph/0203056.

[3] S. Catani, D. de Florian and M. Grazzini, JHEP 0105 (2001) 025; R. V. Harlander and W. B. Kilgore, Phys. Rev. D 64 (2001) 013015, Phys. Rev. Lett. 88 (2002) 201801; C. Anastasiou and K. Melnikov, Nucl. Phys. B 646 (2002) 220; V. Ravindran, J. Smith, W. L. van Neerven, Nucl. Phys. B 665 (2003) 325.

[4] C. Anastasiou, K. Melnikov and F. Petriello, Phys. Rev. Lett. 93 (2004) 262002, hep-ph/0501130.

[5] R. K. Ellis, I. Hinchliffe, M. Soldate and J. J. van der Bij, Nucl. Phys. B 297 (1988) 221; U. Baur and E. W. Glover, Nucl. Phys. B 339 (1990) 38.

[6] D. de Florian, M. Grazzini and Z. Kunszt, Phys. Rev. Lett. 82 (1999) 5209.

[7] V. Ravindran, J. Smith and W. L. Van Neerven, Nucl. Phys. B 634 (2002) 247.

[8] C. J. Glosser and C. R. Schmidt, JHEP 0212 (2002) 016.

[9] S. Catani et al., hep-ph/0005025, in the Proceedings of the CERN Workshop on Standard Model Physics (and more) at the LHC, eds. G. Altarelli and M.L. Mangano (CERN 2000-04, Geneva, 2000), and references therein.

[10] S. Catani, E. D’Emilio and L. Trentadue, Phys. Lett. B 211 (1988) 335.

[11] R. P. Kauffman, Phys. Rev. D 45 (1992) 1512.

[12] D. de Florian and M. Grazzini, Phys. Rev. Lett. 85 (2000) 4678, Nucl. Phys. B 616 (2001) 247.

[13] S. Catani, D. de Florian and M. Grazzini, Nucl. Phys. B 596 (2001) 299.

[14] G. Bozzi, S. Catani, D. de Florian and M. Grazzini, Phys. Lett. B 564 (2003) 65

[15] G. Bozzi, S. Catani, D. de Florian and M. Grazzini, preprint LPSC 05-63, hep-ph/0508068.

[16] C. Balazs and C. P. Yuan, Phys. Lett. B 478 (2000) 192; E. L. Berger and J. w. Qiu, Phys. Rev. D 67 (2003) 034026; A. Kulesza and W. J. Stirling, JHEP 0312 (2003) 056; A. Kulesza, G. Sterman, W. Vogelsang, Phys. Rev. D 69 (2004) 014012; A. Gawron and J. Kwiecinski, Phys. Rev. D 70 (2004) 014003; G. Watt, A. D. Martin and M. G. Ryskin, Phys. Rev. D 70 (2004) 014012 [Erratum-ibid. D 70 (2004) 079902]; A. V. Lipatov and N. P. Zotov, hep-ph/0501172.

[17] http://arturo.fi.infn.it/grazzini/codes.html

[18] A. D. Martin, R. G. Roberts, W. J. Stirling and R. S. Thorne, Phys. Lett. B 604 (2004) 61. 